\title{
Levantamento epidemiológico de cárie dentária em escolares de 5 e 12 anos de idade do município de Cascavel, PR
}

\author{
Epidemiological survey of dental caries in \\ children with 5 and 12 years of age of Cascavel, PR
}

\author{
Marina Berti ${ }^{1}$, Denise de Lima Costa Furlanetto ${ }^{1}$, Marli Maria Schmitt Walker², \\ Mariângela Monteiro de Melo Baltazar ${ }^{1}$, Fabio José Bianchi
}

\begin{abstract}
Resumo
Por solicitação da divisão de saúde bucal da Secretaria Municipal de Saúde de Cascavel-PR, o curso de odontologia da Universidade Estadual do Oeste do Paraná (UNIOESTE) executou o primeiro levantamento epidemiológico na área de Saúde Bucal. A pesquisa foi realizada em crianças nas idades de 5 e 12 anos e foi utilizado os índices epidemiológicos ceo e CPO-D das escolas estaduais e municipais escolhidas através de sorteio contemplando todas as regiões do município. Os resultados obtidos através do levantamento produziram informações sobre as condições de saúde bucal da população de Cascavel. O ceo teve média de 2,42 e o CPO-D foi de 1,91, sendo maior na região Central. Embora os resultados apresentem valores menores que os do SB BRASIL 2000, o trabalho foi de grande valia para a criação e manutenção de uma base de dados contribuindo na perspectiva da estruturação de um sistema de vigilância epidemiológica em saúde bucal.
\end{abstract}

Palavras-chave: cárie dentária; epidemiologia; índice CPO.

\begin{abstract}
At the request of the Division of Oral Health of the Municipal Health Department of Cascavel-PR, the Dentistry course at the Universidade Estadual do Oeste do Paraná (UNIOESTE) performed the first epidemiological survey in the area of Oral Health. The survey was conducted in children aged 5 and 12 years and was used epidemiological indices dtmf and DMFT indexes of state and municipal schools chosen by lottery covering all regions of the city. The results obtained through the survey yielded information on the oral health status of the population of Cascavel. The dtmf had a mean of 2.42 and DMFT was 1.91, being higher in the Central region. Although the results showed lower values than those of SB BRASIL 2000, the work was valuable for the creation and maintenance of a database contributing the perspective of designing a surveillance system in oral health. Keywords: dental caries; epidemiology; DMFT index.
\end{abstract}

Trabalho realizado na Universidade Estadual do Oeste do Paraná (UNIOESTE) - Cascavel (PR), Brasil. 1Professora Associada da Disciplina de Saúde Coletiva da Universidade Estadual do Oeste do Paraná (UNIOESTE) - Cascavel (PR), Brasil. ${ }^{2}$ Especialista e Mestre em Odontopediatria pela Faculdade de Odontologia e Centro de Pesquisas Odontológicas São Leopoldo Mandic - São Paulo (SP), Brasil. ${ }^{3}$ Professor Adjunto da Disciplina de Saúde Coletiva da UNIOESTE - Cascavel (PR), Brasil, e do Centro Universitário de Maringá - Maringá (PR), Brasil. Endereço para correspondência: Fabio José Bianchi - Colegiado de Odontologia do Centro de Ciências Biológicas e da Saúde da Universidade Estadual do Oeste do Paraná - Rua Universitária, 1.619 - CEP: 85814-110 - Cascavel (PR), Brasil - E-mail: fabiojbianchi@yahoo.com.br Conflito de interesses: nada a declarar. 


\section{INTRODUÇÃO}

Em 1986, o Ministério da Saúde (MS) executou o primeiro levantamento epidemiológico de âmbito nacional na área de Saúde Bucal, que foi realizado na zona urbana de 16 capitais, representativo das cinco regiões brasileiras. A pesquisa foi realizada em crianças, adolescentes, adultos e idosos obtendo dados relativos à cárie dentária, à doença periodontal e ao acesso a serviços ${ }^{1}$. Dez anos após, em 1996, foi realizado o segundo levantamento epidemiológico nas 27 capitais brasileiras, na população de 6 a 12 anos, gerando dados relativos à cárie dentária ${ }^{2}$ No ano 2000, o MS iniciou a discussão sobre a realização de um amplo projeto de levantamento epidemiológico que avaliasse os principais agravos em diferentes grupos etários e que incluísse tanto população urbana como rural.

O projeto em questão foi identificado como "SB Brasil: Condições de Saúde Bucal na População Brasileira" e teve início em 1999 com a criação do comitê responsável pela elaboração e execução do mesmo ${ }^{3}$. Em 2001, foi realizado um estudo-piloto, em duas cidades de diferentes portes populacionais - Canela-RS e Diadema-SP. Após a validação da metodologia e dos instrumentos desenhados para o estudo, foi realizado o sorteio dos municípios amostrais e definido o início das oficinas de sensibilização nas diferentes regiões ${ }^{4}$. As oficinas de treinamento e calibração das equipes foram realizadas nos anos de 2002 e 2003, após as quais foi iniciado o trabalho de campo, com realização dos exames e entrevistas. O estudo envolveu a participação de várias instituições e entidades odontológicas - Conselho Federal e Regionais de Odontologia, Associação Brasileira de Odontologia e suas seções regionais, faculdades de Odontologia, além do decisivo de suporte das Secretarias Estaduais e Municipais de Saúde. Aproximadamente 2.000 trabalhadores (cirurgiões-dentistas, auxiliares e agentes de saúde, dentre outros) de 250 municípios estiveram envolvidos na realização do estudo. Sem considerar os ajustes necessários com vista à expansão das taxas para a população em geral, a amostra obtida permite a produção de estimativas segundo as principais variáveis de estratificação previstas no projeto. A amostra pesquisada torna os dados representativos por macrorregião do estado e por porte populacional das cidades envolvidas, além do próprio município para alguns agravos e faixas etárias. Este projeto nacional foi concluído em 2003 e o relatório apresentado com dados do levantamento efetuado fornece informações relativas ao perfil de saúde bucal no Brasil ${ }^{4}$. Trata-se de um relatório descritivo, em que se apresentam os principais resultados obtidos no estudo e definidos de acordo com os objetivos do projeto.

No ano de 2010, a divisão de saúde bucal da Secretaria de Saúde do Município de Cascavel solicitou à disciplina de Odontologia em Saúde Coletiva da Universidade Estadual do Oeste do Paraná
(UNIOESTE) um levantamento epidemiológico que pudesse traçar um perfil dos agravos de saúde bucal da população de Cascavel que desse suporte a um planejamento de ações de prevenção e recuperação da saúde bucal.

$\mathrm{Na}$ impossibilidade de realizar um levantamento que contemplasse toda a população do município nas idades-índice optou-se por indivíduos com idade de 5 anos, por ser essa idade de interesse em relação aos níveis de doenças bucais na dentição decídua, uma vez que podem exibir mudanças em um período de tempo menor que a dentição permanente em outras idades-índice ${ }^{5} \mathrm{e}$ aos 12 anos por ser a idade de monitoramento global da cárie para comparações internacionais e o acompanhamento das tendências da doença ${ }^{6}$.

É de suma importância o estabelecimento da realidade social e epidemiológica de uma população para que, uma vez traçado o perfil e estabelecidas as necessidades das diferentes faixas etárias, sejam viabilizados além do planejamento de ações de promoção de saúde bucal, a avaliação das mesmas ${ }^{5}$. A impossibilidade de contemplar todas as faixas etárias em um primeiro momento nos levou à priorização de duas faixas etárias inicialmente, as quais permitissem uma avaliação da dentição decídua e permanente.

Este trabalho teve por objetivo estimar, para a população de escolares de 5 e 12 anos do município de Cascavel, a prevalência de cárie dentária, a fim de subsidiar pesquisas que visam ao estabelecimento de relações entre os dados encontrados e a realidade socioeconômica e demográfica da população de Cascavel. Através do conhecimento do quadro epidemiológico de cárie dentária nos escolares da amostra deste município propor à SMS estratégias para enfrentamento do problema.

\section{MÉTODOS}

O processo de amostragem adotado neste estudo foi amostragem por conglomerado (duplo estágio) e o estudo foi do tipo transversal. Inicialmente o município foi dividido em quatro macrorregiões (Norte, Sul, Leste e Central). Em seguida, as escolas foram divididas segundo seu tamanho: pequeno, médio e grande porte. Este procedimento é preconizado a fim de tornar a amostra o mais homogênea possível dentro de cada grupo ${ }^{7}$. Ficou estabelecido que escolas de pequeno porte seriam aquelas com até 50 alunos matriculados; de médio porte aquelas entre 51 e 149 alunos; e grande porte com 150 alunos ou mais. Elaborou-se então um sorteio, de modo que 16 escolas de pequeno porte, 11 de médio e 3 de grande porte de cada macrorregião fossem incluídas na amostra, totalizando 30 escolas.

O cálculo do tamanho da amostra foi feito pelo programa Epi-Info, de domínio público ${ }^{7}$ o qual possibilita o cálculo de tamanho mínimo da amostra para estabelecimento de prevalência 
em populações finitas, utilizando-se nível de confiança de $95 \%$. O número total de crianças determinadas para inclusão foi de 1950, dos quais 405 alunos eram pertencentes às escolas de pequeno porte; 991 , de médio porte e 554 , de grande porte. Um total de 1.014 crianças de 5 anos e 936 crianças de 12 compuseram a amostra final. Foram adotados como critério de inclusão que os escolares apresentassem a idade de estudo e que seus responsáveis legais aceitassem através da assinatura do Termo de Consentimento Livre Esclarecido.

Aproximadamente 100 pessoas, incluindo acadêmicos de odontologia, professores e cirurgiões-dentistas da SMS, participaram do estudo como examinadores, anotadores ou coordenadores, sendo os mesmo liberados de suas atividades rotineiras para a execução do presente trabalho.

Para assegurar uma interpretação uniforme e consistente dos critérios padronizados para a coleta dos dados, os acadêmicos participaram de exercícios de calibração. Foram realizadas oficinas de calibração, com o propósito de calibrar as equipes de campo, minimizando as variações entre os diferentes examinadores.

Os exames intrabucais foram realizados em escolas e creches por equipes especialmente treinadas compostas de um docente cirurgião-dentista e acadêmicos do curso de odontologia. Os examinadores permaneceram na área de exame (pátio da escola ou sala designada pela direção do local) e o anotador conduziu os alunos (em grupos de 10 e de acordo com a lista previamente elaborada) da sala de aula ao local do exame e retorno. Cada equipe utilizou espátulas de madeira e os exames transcorreram respeitando-se as normas de biossegurança ${ }^{7}$.

Os critérios adotados neste estudo tomaram por base a $4^{\mathrm{a}}$ edição do Oral Health Surveys - Basic Methods da Organização Mundial da Saúde ${ }^{8}$ descritos no Manual do Examinador e no Manual do Anotador, produzidos pela equipe de coordenação do Projeto SB Brasil. Assim, foram utilizados os índices de ataque de Cáries - CPO-D para a dentição permanente e o ceo-d para a dentição decídua. Ambos os índices são utilizados para medir e comparar a experiência de cárie dentária, sendo que seu valor expressa a média de dentes cariados, perdidos e obturados em um indivíduo e/ou grupo de indivíduos. Através do registro das necessidades de tratamento, podem também ser identificadas, além de necessidades propriamente ditas, a presença de lesões não cavitadas (mancha branca presente) e os diferentes níveis da doença ativa (cárie de esmalte, cárie de dentina e cárie próxima à polpa). Deste modo, uma maior qualificação do índice pode ser proporcionada pela verificação das necessidades de tratamento. Foram observadas as seguintes características relativas à saúde bucal: coroa dentária e necessidade de tratamento odontológico do elemento dentário.
Os dados foram tabulados e analisados através do programa estatístico Graph Pad Prism R com nível de significância de 5\%9.

O presente trabalho foi aprovado pelo Comitê de Ética e Pesquisa da UNIOESTE sob o protocolo de número 169/2008.

\section{RESULTADOS}

Foram examinados 586 escolares de 12 anos e 699, de 5 anos de idade. Dentre os indivíduos com 12 anos de idade, as médias do índice CPO-D apresentadas nas regiões sul, norte, leste e central foram respectivamente 1,$58 ; 1,98 ; 1,4$ e 2,63 totalizando um CPO-D aos 12 anos de 1,91. Os componentes "cariado" e "restaurado" representaram respectivamente $4,18 \%$ (607) e 3,38\% (491) enquanto que os hígidos representaram 92,44\% (13.389) do total de dentes examinados (15.629).

Com relação ao ceo-d foram observados as seguintes médias de acordo com as regiões sul, norte, leste e central 2,66; 3,16; 2,02 e 2,16, respectivamente, totalizando um ceo-d aos 5 anos de 2,42 . Os componentes "cariado" e "restaurado" representaram respectivamente $9,92 \%$ (1.346) e 2,43\% (330) enquanto que os hígidos representaram $87,65 \%$ (11.830) do total de dentes examinados (13.564).

\section{DISCUSSÃO}

O resultado desse estudo foi mais favorável do que o encontrado no levantamento das condições de saúde bucal da população brasileira (Projeto SB Brasil) cuja média do índice CPO-D aos 12 anos em 2003 foi de 2,8 e da região sul foi de 2,31. Este resultado representa também o alcance da meta proposta pela OMS de CPO-D menor que 3 nas crianças de 12 anos, permitindo estabelecer que a doença cárie pode ser considerada controlada no município.

Foi observado que o CPO-D das regiões menos favorecidas economicamente da cidade como a região Norte apresentou média maior que as demais. Nesta, engloba os bairros Canadá, Cancelli, Clarito, Claudete, Floresta, Independência, Interlagos e Jardim Brasmadeira. Maltz e Silva, em 2001 ${ }^{10}$, relacionaram o grau de instrução dos pais e prevalência de cárie em adolescentes de 12 anos de idade na cidade de Porto Alegre e observaram uma relação positiva entre grau de instrução dos pais e experiência de cárie, tendo maior necessidade de tratamento voluntários estudantes de escolas públicas. Esses autores, também observaram o fenômeno da polarização da doença ${ }^{10}$. A situação de saúde bucal brasileira está intimamente relacionada com as grandes desigualdades socioeconômica vigente e o difícil acesso à assistência odontológica ${ }^{11}$. 
A região sul e leste da cidade apresentaram médias de CPO-D compatíveis com países desenvolvidos como Noruega ${ }^{12}$. Nestas, há um maior poder aquisitivo e desta forma a procura por serviços especializados torna-se mais fácil.

Gushi et al. ${ }^{13}$ apresentaram a relação entre fatores socioeconômicos e cárie dental entre adolescentes, sendo que quanto mais desfavoráveis as condições socioeconômicas maior foi a experiência de cárie.

Alguns autores consideram a situação socioeconômica como um fator determinante do risco de cárie, caracterizando uma doença de países considerados em desenvolvimento ${ }^{12-14}$.

A região central apresentou número elevado do CPO-D em virtude de ser uma mistura dos vários bairros que foram enquadrados na análise, pois a mesma acaba sendo uma referência em educação. Desta forma, não há limitação de estudantes que residem na região central.

Com relação ao ceo-d avaliado em escolares de 5 anos de idade foi observado um comportamento semelhante ao CPO-D. As regiões que apresentaram índices maiores foi centro e norte. Na região central estão alocados crianças de diversos bairros que acabam sendo mais vantajosos por estar próximo ao trabalho da mãe, não refletindo a relação setorial dos bairros. Assim como no CPO-D a região norte que apresenta bairros com menores condições socioeconômicas apresentaram maior média deste componente, reforçando a importância do nível socioeconômico com número de dentes afetados por cáries.

O índice ceo-d encontrado aos 6 anos de idade foi de 2,42 (média ponderada entre escolares da rede pública e privada), e a percentagem de crianças livres de cárie foi de 52,2\%.

\section{CONSIDERAÇÕES FINAIS}

Através do levantamento foi possível produzir informações sobre as condições de saúde bucal da população alvo do estudo a fim de subsidiar o planejamento/avaliação de ações nessa área no nível da SMS, bem como a criação e manutenção de uma base de dados relativa aos principais problemas nesse campo, contribuindo na perspectiva da estruturação de um sistema de vigilância epidemiológica em saúde bucal.

\section{REFERÊNCIAS}

1. Brasil. Ministério da Saúde - Divisão Nacional de Saúde Bucal Levantamento Epidemiológico em Saúde Bucal: Brasil, zona urbana. Brasília; 1986. 137p.

2. Brasil. Ministério da Saúde. Secretaria de Assistência à Saúde. Departamento de Assistência e Promoção à Saúde. Coordenação de Saúde Bucal. Levantamento Epidemiológico em Saúde Bucal: $1^{\text {a }}$ etapa cárie dental - projeto. Brasília; 1996.

3. Brasil. Ministério da Saúde. SB 2000: Condições de Saúde Bucal na População Brasileira no ano 2000. Brasília; 2000.

4. Roncali AG, Frazão P, Patussi MP, Araújo IC, Ely HC, Batista SM. Projeto SB 2000: uma perspectiva para a consolidação da epidemiologia em saúde bucal coletiva. RBO. 2000;1(2):9-25.

5. Departamento de Atenção Básica. Secretaria de Atenção à Saúde, Ministério da Saúde. Projeto SB Brasil 2003: condições de saúde bucal da população brasileira 2002-2003. Resultados principais. Brasília: Ministério da Saúde; 2004.

6. Pine CM, Pitts NB, Nugent ZJ. British Association for the Study of Community Dentistry (BASCD) guidance on sampling for surveys of child dental health. A BASCD co-ordinated dental epidemiology programme quality standard. Community Dent Health. 1997;14(1):10-7.

7. Dean AG, Dean JA, Coulombier D, Brendel KA, Smith DC, Burton $\mathrm{AH}$, et al. Epiinfo Ver 6: A word processing, database and statistics programme for epidemiology on microcomputers. Atlanta, Georgia, USA: Centre for Disease Control and Prevention; 1994. Disponível em: http://www.cdc.gov/epiinfo/Epi6/ei6.htm
8. WHO. World Health Organization. Oral Health Surveys. Basic Methods. Geneva: WHO, 1997.

9. Santos AC, Ladoninsky LH, Tochetto LRA, Carneiro JP, Oriente A, Bianchi FJ, et al. Densidade populacional dos neurônios mioentéricos da curvatura gástrica maior do estômago glandular de ratos induzidos ao alcoolismo crônico. Saúde e Pesquisa. 2010;3:285-9.

10. Maltz M, Silva, BB. Relação entre cárie, gengivite e fluorose e nível socioeconômico em escolares. Rev Saúde Pública. 2001;35(2):170-6.

11. Baltazar MMM, Giordani MT, Furlanetto DLC, Berti M, Bianchi FJ. Levantamento das Condições de Saúde Bucal de Crianças e Adolescentes das Comunidades Rurais Atendidas em Unidade Móvel de Saúde no Oeste do Paraná. UNOPAR Cient Ciênc Biol Saúde. 2011;13(4):257-61

12. Koposova N, Eriksen HM, Widström E, Handegård BH, Pastbin M, Koposov R. Caries prevalence and determinants among 12-yearolds in North-West Russia and Northern Norway. Stomatologija. 2013;15(1):3-11.

13. Gushi LL, Soares Mda C, Forni TI, Vieira V, Wada RS, Sousa Mda L. Cárie dentária entre os adolescentes e sua relação com as variáveis sócioeconômicas. J Appl Oral Sci. 2005;13(3):305-11.

14. Moysés SJ. Desigualdades em saúde bucal e desenvolvimento humano: um ensaio em preto, branco e alguns tons de cinza. RBO. 2000;1:19-28.

Recebido em: 03/09/2012 Aprovado em: 31/10/2013 